\title{
Mechanical versus biological aortic valve replacement strategies
}

\section{Reineke, F Gisler, L Englberger \& T Carrel}

To cite this article: D Reineke, F Gisler, L Englberger \& T Carrel (2015): Mechanical versus biological aortic valve replacement strategies, Expert Review of Cardiovascular Therapy, DOI: 10.1586/14779072.2016.1133293

To link to this article: http://dx.doi.org/10.1586/14779072.2016.1133293

Accepted author version posted online: 17

Dec 2015.

Submit your article to this journal

Џll Article views: 9

Q View related articles $\asymp$

View Crossmark data $\asymp$ 
Publisher: Taylor \& Francis

Journal: Expert Review of Cardiovascular Therapy

DOI: $10.1586 / 14779072.2016 .1133293$

\title{
Mechanical versus biological aortic valve replacement strategies
}

\author{
D Reineke, Gisler F, Englberger L, Carrel T
}

University Hospital Berne

Bern $\mathrm{CH}-3010$

Switzerland

Corresponding Author:

Thierry Carrel

University Hospital Bern

Bern

Switzerland

thierry.carrel@insel.ch

\section{Abstract}

Aortic valve replacement (AVR) is the most frequently performed procedure in valve surgery.

The controversy about the optimal choice of the prosthetic valve is as old as the technique itself. Currently there is no perfect valve substitute available. The main challenge is to choose between mechanical and biological prosthetic valves. Biological valves include pericardial (bovine, porcine or equine) and native porcine bioprostheses designed in stented 
or stentless versions. Homografts and pulmonary autografts are reserved for special indications and will not be discussed in detail in this review. We will focus on the decision making between artificial biological and mechanical prostheses, respectively. The first part of this article reviews guideline recommendations concerning the choice of aortic prostheses in different clinical situations while the second part is focused on novel strategies in the treatment of patients with aortic valve pathology.

Keywords: aortic valve replacement, biological valve prosthesis, mechanical valve prosthesis, oral anticoagulation, novel oral anticoagulants.

\section{Current Evidence}

The current guidelines of the European Society of Cardiology from 2012 [1] and of the American Heart Association from 2014 [2] uniformly recommend mechanical aortic valve replacement $(A V R)$ in patients under 60 years of age and biologic AVR in patients over 70 years of age (Fig. 1). In patients between 60 and 70 years of age, recommendations are conflicting. The ESC-Guidelines recommend biologic prosthesis from the age of 65 years onwards, whereas the newer AHA/ACC guidelines only recommend biological valves starting with 70 years of age. Looking at the development of the guidelines over the last 20 years there is a shift away from a clear-cut age limit towards the patients wish and life-style considerations.

Currently there is a trend towards more biological AVR, also in patients under 65 years of age, which is contrary to the progress of life expectancy of patients at this age.

Justification for this approach is the option for a valve-in-valve procedure in the case of structural valve deterioration (SVD) which might become a routine bail out strategy. In addition, the last generation of pericardial tissue valves may have excellent long-term durability over 20 years. However, current studies also demonstrate a significant age- 
dependency in durability with lower rates of SVD when implanted in increased age $[3,4]$. The conclusion to implant biological valves at a younger age could therefore be contraproductive. Another limitation is, that several valves on market show significant shorter longterm results with SVD already in less than 10 years after implantation.

In literature, most clinical studies demonstrate higher rates of reoperation and decreased survival after biological AVR compared to mechanical AVR.

Bourguignon et al. identified both the cumulative risk of reoperation due to SVD and the patient's age at surgery as the unique covariate for survival [3].

With future life expectancies of $>85$ years, prosthetic valve durability of at least 15 to 20 years will be needed in a 65 years old patient in order to avoid re-interventions. The probability of reoperation due to SVD after 20 years in a 65 years old patient is higher than $15 \%[3]$.

The ad hoc argument for the use of biological valves in younger patients is often the possibility of valve-in-valve procedures, when SVD occurs. However, this is not justified by data, yet. Larger clinical studies are rare in this field and the reports present only a limited number of prostheses types [5-7]. In addition, the large Valve-in-valve registry demonstrates a high rate of patient-prosthesis mismatch, especially when implanted in a valve smaller than 25mm [8] Valve-in-valve procedures should therefore only be performed under studyprotocol or registries to analyze long-term results.

One principal problem is the lack of long-term data after transcatheter aortic valve implantation (TAVI). There are only few studies available on durability or rate of SVD with follow-up times beyond 5 years $[9,10]$. At this time, it is unclear if transcatheter valves have durability comparable with the last generation of surgical valve prostheses, and some reports raise doubts. With a 5-year overall mortality of around $50 \%$ after transcatheter valve implantation, it will be difficult to show a non-inferiority in durability compared with surgical 
implanted biologic valves, because of a low number of survivors in TAVI cohorts.

The major limitation with mechanical prostheses is the required life-long anticoagulation with subsequent complications. Valve thrombosis, thromboembolic events and bleeding events account for $75 \%$ of all complications that occur after mechanical heart valve replacement.

In the current guidelines, antiplatelet agents are recommended additionally to vitamin $\mathrm{K}$ antagonists (VKA) as a protective factor against thromboembolic events [2,11]. There was a reduction in thromboembolic events in patients with additional antiplatelet therapy. Several studies showed that the risk of thromboembolic events are similar between biological and mechanical valve prostheses and can be reduced by administering antiplatelet agents [1214]. Self-management of oral anticoagulation with self-measuring of the INR (international normalized ratio) may reduce the rate of thromboembolic events, but showed no difference in bleeding complications $[15,16]$.

New oral anticoagulants are not applicable in patients with valve prosthesis. Studies had to be terminated due to adverse outcome with higher rates of thromboembolic events and higher risk of bleeding compared to standard warfarin therapy [17].

Furthermore, the selection of the valve prosthesis depends on various patient-related factors, such as age at implantation, compliance with long-term anticoagulation, risk factors for and history of bleeding events, life expectancy, life-style and the patient's preferences.

Concerning the need for anticoagulation after valve replacement with a mechanical valve, it has to be taken into account that a substantial number of patients after biological valve replacement need oral anticoagulation as well by other indications mainly due to the onset of atrial fibrillation. In a prospective study of over 6000 participants, lifetime risk to develop AF 
at the age of 55 years was already $23.8 \%$ in men and $22.2 \%$ in women. This is increased with concomitant cardiovascular, structural heart disease and age $[18,19]$.

Randomized trials comparing biological and mechanical valve replacements are rare.

Stassano et al. randomized 310 patients between 55 and 70 years of age into a mechanical and a biological prosthesis group to undergo AVR. They found similar mortality and major adverse prosthesis-related events (thromboembolism, bleeding, endocarditis, SVD, nonstructural dysfunction) in the two groups over a mean follow up of just over 4 years [20].

Brown et al. analyzed outcome after AVR with mechanical versus biological prosthesis in patients aged 50 to 70 years at operation. Freedom from reoperation at 10 years was $98 \%$ for mechanical valves and $91 \%$ for bioprostheses $(p=0.06)$ [21]. Rates of late stroke or other embolic events and of endocarditis were similar between the two groups. Rehospitalisation because of hemorrhagic events occurred in $15 \%$ of patients with mechanical valves and $7 \%$ of patients with bioprostheses $(p=0.01)$. The 5 - and 10 -year unadjusted survivals were $87 \%$ and $68 \%$ for mechanical valves and $72 \%$ and $50 \%$ for bioprostheses, respectively $(P<0.01)$. With this superior survival of the patient group after mechanical AVR the authors conclude that there is insufficient evidence to recommend bioprosthetic valves in the aortic position for patients younger than 65 years.

In accordance with this data, Weber et al. conclude from a cohort of patients younger than 60 years old that biologic aortic valve replacement is associated with reduced mid-term survival compared with survival after mechanical aortic valve replacement. Survival was significantly reduced in patients after biologic aortic valve replacement $(90.3 \%$ vs $98 \%$; $P=$ $0.038)[22]$. 
The latest trial by a Swedish group showed the same results with better long-term survival in patients aged 50-69 years after AVR with mechanical valves compared with those who had received bioprostheses. Additionally they analyzed age subgroups and showed a significant difference in cumulative survival in the subgroup 50-59 years compared with no significant difference in the age group 60-69 years [23].

\section{Infective endocarditis}

Infective endocarditis (IE) has a high mortality rate, even with appropriate antibiotic and surgical therapy. The risk of IE is 50 times higher in patients with a prosthetic valve compared with the general population. In developed countries, IE is most often associated with prosthetic valves and intracardiac devices. Prosthetic valve endocarditis (PVE) is reported to occur with a $0.3 \%$ to $1.2 \%$ patient-year incidence. It accounts for $10-30 \%$ of all cases of IE. [2].

Both mechanical and bioprosthetic valves can be involved by the infection and their rate of infection is similar at 1 year. In the long-term biological prostheses seem to have a higher incidence of PVE compared to mechanical prostheses [24]. This was again confirmed in a recent study by Brennan et al. They reported a 12-year incidence of rehospitalisation for endocarditis of $2.2 \%$ for patients with bioprosthesis versus $1.4 \%$ after mechanical AVR . This has been explained by the fact that a degenerating valve alone presents a risk factor for PVE [25].

In patients suffering from IE the optimal timing and the choice of substitute - homograft, biological or mechanical valve prosthesis - is complex and studies are conflicting. Operative 
mortality is similar in mechanical and biological AVR [1]. The 2 to $3 \%$ risk of reinfection during valve replacement is not as high as the mortality rate of IE without surgical therapy which is as high as $51 \%$ [26].

Nguyen et al. compared a total of 167 patients with IE undergoing AVR with biological (18\%), mechanical prosthesis $(65 \%)$ or with a homograft $(16 \%)$. They found a lower 5 -year survival with bioprosthesis than after AVR with mechanical prosthesis in patients up to 65 years of age. However, no significant difference between the two groups was present in patients over 65 years of age. Homografts showed no difference from mechanical valves in both age groups [27].

Moon et al. reported in an epidemiological study a rate of recurrent or residual endocarditis of $1.5 \%$ per patient-year for native valve endocarditis and $2.5 \%$ for prosthetic valve endocarditis. There was no significant difference in endocarditis rates between mechanical $(1.2 \%)$ and bioprosthetic $(1.8 \%)$ valve substitutes. Operative mortality and complication-free survival were also similar within the two valve types [28].

Based on this data we recommend to replace infected valves, whether native or prosthetic, with a mechanical prosthesis in younger patients and with a biological prosthesis in patients older than 70 years or with limited life expectancy as recommended in the AHA guidelines for normal AVR without any infection. Between 60 and 70 years of age, it depends on other risk factors, bleeding complications in the history and life expectancy. 


\section{Bleeding}

Mechanical heart valves produce abnormal flow with areas of low flow as well as areas with high flow and subsequent high shear stress which can cause platelet activation. To prevent valve thrombosis and to minimize the risk of thromboembolic events, patients with mechanical AVR receive life-long oral anticoagulation therapy with vitamin $\mathrm{K}$ antagonists (VKA). [2,25,29].

When low-dose aspirin (75 to $100 \mathrm{mg}$ daily) is administered additionally to VKA, the risk of major emboli can be reduced from $8.5 \%$ to $1.9 \%(\mathrm{OR} 0.43 ; 95 \% \mathrm{Cl}: 0.32$ to 0.59$)$, the risk of stroke from $4.2 \%$ to $1.3 \%$ and the overall mortality from $7.4 \%$ to $2.8 \%$ (OR $0.57 ; 95 \% \mathrm{Cl}$ : 0.42 to 0.78 ). The risk of major bleeding, however, is increased when antiplatelet agents are added to oral anticoagulants (OR 1.58; 95\% Cl: 1.14 to 2.18 ) [29].

VKA administration with target INR 2-3 was compared with high degree anticoagulation with INR target 3-4. The thromboembolic event rate was not reduced significantly in the high dose group, but the bleeding risk could be reduced significantly with low dose anticoagulation (INR 2-3) [30]. Comparable results were found with additional antiplatelet therapy. The risk of gastro-intestinal irritation or hemorrhage is dose dependent, but the antiplatelet effect is not. The benefit of higher dose aspirin (> 100mg) is not superior to low-dose aspirin (75 to $100 \mathrm{mg}$ ), but the risk of major bleeding is significantly increased in the high-dose group.

Consequently the AHA Guidelines from 2013 recommend oral anticoagulation with target INR of 2.5 (2.0 to 3.0) for mechanical prosthesis in aortic position combined with low-dose aspirin $100 \mathrm{mg}$ daily $[2,29]$. 
The remaining annual risk of thromboembolic events in patients with a mechanical valve prosthesis is $1-2 \%$ compared with $0.7 \%$ with a bioprosthesis.

\section{Valve choice in women of child-bearing age}

Women with a prosthetic heart valve have a higher rate of adverse outcomes than expected in a general obstetric population. This includes maternal mortality, miscarriage, thromboembolism and obstetric hemorrhage. Infants of these women are at anincreased risk of perinatal mortality, preterm birth, small-for-gestational-age and congenital malformations $[31,32]$. The selection of prosthesis is a major challenge in women with the desire to have children. The 2012 ECS-Guidelines emphasized the high risk of thromboembolic complications with a mechanical prosthesis during pregnancy despite correct anticoagulation. A planned pregnancy is considered a class /la and level C evidence [33] for the implantation of a bioprosthesis.

After biological AVR the majority of patients will need a reoperation within the next 8 to 10 years. There are conflicting data of accelerated deterioration of prosthetic heart valves during pregnancy [28]. However. in this very young age group, even without accelerated deterioration by pregnancy, the durability of bioprostheses is limited $[3,20]$. Comparing the mortality of reoperation in this patient population with the overall risk of VKA, there is a mortality of around $6 \%$ for reoperation and around $5 \%$ in patients taking VKA $[31,33]$.

Women with a mechanical valve-prosthesis have two major problems: (1) a hyper-coagulable state exists throughout pregnancy resulting in a higher risk of thromboembolic complications and (2) vitamin $\mathrm{K}$ antagonists (VKA) cross the placenta, increasing the risk of early abortion, embryopathy and prematurity. There are reports of teratogenity of VKA in the first trimester of pregnancy with higher rates of spontaneous abortion under VKA [34]. The incidence of embryopathy is still debated and the teratogenic effect of VKA may be masked by the high rate of miscarriages and therapeutic abortions [35]. During delivery there is a higher risk of intracranial bleeding. Van Hagen et al. compared 212 patients with a mechanical valve with 
134 patients with a tissue heart valve and 2620 patients without a prosthetic valve. Women with a mechanical heart valve had only a $58 \%$ chance of an uncomplicated pregnancy with a live birth [36].

Timing of AVR and pregnancy is an important factor. If $A V R$ is performed with a bioprosthesis, pregnancy should be planned within the next few years because of expected durability of only 7 to 10 years. If there is a severe deterioration of the prosthetic valve with the need for reoperation during pregnancy, it will be a high-risk operation with a fetal mortality of $30 \%$ to $40 \%$ and a maternal mortality rate of up to $9 \%$.

\section{Renal Insufficiency}

Patients with end stage renal disease (ESRD) suffer from chronic uremia, hypertension and hyperlipidemia and need frequent vascular access together with impaired immunity, which lead to a heightened risk of endocarditis. All these factors lead to a high cardiac mortality and morbidity and to a poor survival, with a 5 -year mortality rate of approximately $65 \%[37,38]$. If a patient suffers additionally from diabetes or coronary artery disease, the survival rate is even lower.

Earlier studies reported high bleeding complications in patients with ESRD. Okada et al. showed that the bleeding rate is age dependent with higher rates in older patients. If they were matched in age groups, there were no significant differences in bleeding rates between biological and mechanical prosthesis. But they reported a significant difference in bleeding complications between patients under dialysis versus non-dialysis patients $[38,39]$.

Data on SVD in patients with ESRD are lacking, mainly because of a low survival rate in this patient group. Studies showed low rate of SVD, but with a mean follow-up of only 5 years. Even with accelerated valve failure, this time is too short to demonstrate significant 
differences. An important comorbidity in ESDR is secondary hyperparathyroidism. Okada et al. describe a very strict management of patients with secondary hyperparathyroidism. They performed a parathyroidectomy, when the patient could not be controlled with aggressive medication. Indications for heart valve replacement in patients with ESRD are the same as in patients with normal renal function. But the choice of optimal valve prosthesis is a subject of debate.

Previous guidelines recommended mechanical valve prostheses in patients with ESRD because of the general concern that bioprosthetic valves undergo premature failure caused by accelerated calcification. This concern is not well-founded and there are only few reports which could not be confirmed in newer studies.

Regarding recent studies, the choice between bioprosthetic and mechanical valves can be recommended with the same decision criteria as in patients with normal renal function and should be oriented primarily on age and life expectancy. Whereas a younger patient under dialysis without important comorbidities has a realistic survival rate to experience SVD, a patient in his seventies with diabetes has a poor survival and won't experience SVD even with accelerated valve deterioration caused by increased calcium level. At the same time patients on chronic dialysis tend to have more hemorrhagic events. Therefore, the choice in these patients will be in favor of a biological valve replacement even in patients under the age of 65 years [2,40].

\section{Expert commentary}

The necessity to choose between a mechanical and biological valve is due to the necessity to anticoagulate mechanical valves. The invention of a valve without the need for anticoagulation would alter strategies of valve choice. Despite a long history of success the 
search for the ideal replacement valve is ongoing. The ideal prosthetic valve would combine high performance, lack of turbulence, risk free implantation and no maintenance. Present tissue valves fall short of these ideals as $15 \%$ will fail after 20 years in patients receiving them at the age of 65 years [3]. The inconvenience of warfarin and the annual risk of bleeding (1-2\%) make mechanical valves also fall short of this ideal [29].

The medical industry is not only innovative in biological valve design, but also in mechanical valve prostheses. There are some new designs available. The main goal will be to reduce thrombogenity, so that it would be possible to reduce INR targets. The biggest challenge will be, not to pay these lower INR-targets with higher rates of thromboembolic events.

With the On-X aortic heart valve (Fig. 2) a valve prosthesis with recommended lower INRtarget is available. Very recently, the On-X mechanical heart valve (On-X Life Technology Inc.) has received U.S. FDA clearance for an expanded labeling claim that allows patients with On-X aortic heart valves to be managed at an INR level of 1.5 to 2.0 , the lowest rate for any other mechanical valve. Patients experienced a $65 \%$ overall reduction in bleeding events with no increase in stroke rate [41].

The Lapeyre-Triflo FURTIVA ${ }^{\circledR}$ is similar to the On-X aortic valve a further step into finding the ideal mechanical prosthesis (Fig. 3). Due to an innovative anatomic tri-leaflet design with a physiological operating mode and being composed of high-performance biocompatible materials, FURTIVA ${ }^{\circledR}$ may be the first prosthetic heart valve resistant to both thrombosis and

structural failure which at the same time functions without warfarin anticoagulation therapy [42].

New mechanical valve types will also have their effect on the treatment on women of childbearing age. Only limited data exists on outcomes of contemporary pregnancies in the setting of maternal heart valve prostheses. Most of the reviews are still based on 
pregnancies from the previous four decades including ball-and-cage valves with high anticoagulation management $[43,44]$.

A recent review by Lawley et al. [32] is the first to investigate these questions in a contemporary setting defined as the last 20 years. The current review showed that no deaths occurred due to valve thrombosis reflecting improvement in prosthesis design and management. Although outcomes have improved considerably the main question is still left unanswered. How does one balance the increased risk of mortality and thrombosis during pregnancy in women with mechanical valves against the need for more frequent valve replacement with bioprosthetic devices? One can only speculate that modified anticoagulation regimes and new valve types like the above mentioned On-X and Lapeyre Triflo FURTIVA ${ }^{\circledast}$ might push the balance toward a mechanical AVR in women of childbearing age in the future.

When implanting mechanical valves most surgeons promise their patients that in the near future there will be patient friendly alternatives to warfarin. Novel oral anticoagulants like dabigatran (direct thrombin inhibitor) where thought to fulfill these promises as their use in patients with atrial fibrillation and animal studies suggested a potential role in patients with mechanical heart valves.

The RE-ALIGN study was the first randomized study to validate a dabigatran dosing regimen for the prevention of thrombosis in mechanical heart valve patients. The trial was prematurely terminated due to excess bleeding events in patients receiving dabigatran. It was not as effective as warfarin for the prevention of thromboembolic complications [17].

Whether other novel anticoagulants such as rivaroxaban and apixaban (factor Xa inhibitors) would result in better outcomes compared with dabigatran needs to be investigated.

It has to be emphasized that none of the current valves available can fully restore native valve function due to their lack of growth and remodeling capabilities. Heart valve tissue engineering tries to overcome these limitations. Trileaflet heart valve tissue engineering aims to develop valve substitutes in vitro or situ, by using the regenerative potential of the body for 
the tissue culture phase. The journey to a truly autologous tissue-engineered heart valve is still been a long one but there is very promising progress $[45,46]$.

\section{Five-year view}

The next five years will mainly be marked by the development of TAVI. If the industry is able to reduce pacemaker rates and paravalvular leakage and studies are able to present freedom from structural valve degeneration of over 10 years, TAVI will most surely become an option for younger patients and therefor will influence the choice between biological valves and mechanical valves in view of the valve-in-valve option.

These trends would surely be influenced by the development and launch of an anticoagulation free mechanical valve and suitable NOAKs. The goal in the end will be to provide every patient with one life-long solution without having to worry about valve degeneration, thrombosis and re-intervention.

\section{Key issues}

- Current guidelines recommend mechanical aortic valve replacement (AVR) in patients under 60 years of age and biological AVR in patients over 70 years of age. In patients between 60 and 70 years of age, recommendations are conflicting.

- Looking at the development of the guidelines over the last 20 years there is a shift away from a clear-cut age limit towards the patients wish and life-style considerations.

- The probability of reoperation due to structural valve deterioration of a tissue valve after 20 years in a 65 years old patient is $>15 \%$. 
- Valve-in-valve treatment is still experimental and should carefully be addressed in the decision-making progress when it comes to implanting biological valves at an earlier age.

- In the current guidelines, antiplatelet agents are recommended additionally to vitamin $\mathrm{K}$ antagonists as a protective factor against thromboembolic events.

- Both mechanical and bioprosthetic valves can be involved by the infection and their rate of infection is similar at 1 year. In the long-term biological prostheses seem to have a higher incidence of PVE compared to mechanical prostheses.

- The annual risk of thromboembolic events in patients with a mechanical prosthesis is $1-2 \%$ versus $0.7 \%$ with a bioprosthesis even with appropriate antithrombotic therapy.

- Women with a prosthetic heart valve have a higher rate of adverse outcomes than expected in a general obstetric population.

- In patients with renal insufficiency the choice of valve type can be made with the same decision criteria as in patients with normal renal function.

- New mechanical prostheses allow lower INRs and, one day, maybe no anticoagulation at all. The biggest challenge will be, not to pay these lower INRtargets with higher rates of thromboembolic events.

- Modified anticoagulation regiments and new valve types might in the future push the balance toward a mechanical AVR in in women of child-bearing age.

- The use of novel oral anticoagulants in patients with mechanical heart valves should be avoided at this time.

\section{Financial and competing interests disclosure}

The authors have no relevant affiliations or financial involvement with any organization or entity with a financial interest in or financial conflict with the subject matter or materials 
discussed in the manuscript. This includes employment, consultancies, honoraria, stock ownership or options, expert testimony, grants or patents received or pending, or royalties.

\section{References}

Reference annotations

* Of interest

** Of considerable interest

${ }^{*}[1]$ Vahanian A, Alfieri O, Andreotti F et al. Guidelines on the management of valvular heart disease (version 2012). Eur Heart J 2012;33:2451-96

Guidelines of the European Society of Cardiology (ESC) and the European Association for Cardiothoracic Surgery (EACTS) on the management of Valyular heart disease from 2012

**[2] Nishimura RA, Otto CM, Bonow RO et al. 2014 AHA/ACC Guideline for the Management of Patients With Valvular Heart Disease: a report of the American College of Cardiology/American Heart Association Task Force on Practice Guidelines. Circulation 2014;129:521-643

Guidelines of the American Heart Association (AHA) and the American College of Cardiology (ACC) on the management of Valvular Heart Disease from 2014

*[3] Bourguignon T, Bouquiaux-Stablo A-L, Candolfi P et al. Very Long-Term Outcomes of the Carpentier-Edwards Perimount Valve in Aortic Position. Ann Thorac Surg 2015;99:831-7 Long-Term follow-up of patients with aortic bioprosthesis analyzed according to age. They show good long-term results after 20 years in patients of 70 years of age at replacement, but show early SVD when implanted in younger patients. 
[4] Johnston DR, Soltesz EG, Vakil N et al. Long-Term Durability of Bioprosthetic Aortic Valves: Implications From 12,569 Implants. Ann Thorac Surg 2015;99:1239-47

[5] Di Eusanio M, Saia F, Pelliciari G, et al. In the era of the valve-in-valve: is transcatheter aortic valve implantation (TAVI) in sutureless valves feasible? Ann Cardiothorac Surg $2015 ; 4: 214-17$

[6] Duncan A, Davies S, Di Mario C, et al. Vavle-in-valve transcatheter aortic valve implantation for failing surgical aortic stentless bioprosthetic valves: A singlee-center experience. J Thorac Cardiovasc Surg 2015;150:91-8

[7] Gurvitch R, Cheung A, Ye J, et al. Transcatheter valve-in-valve implantation for failed surgical bioprosthetic valves. J Am Coll Cardiol 2011;58:2196-209

*[8] Conradi L, Silaschi M, Seiffert M, et al. Transcatheter valve-in-valve therapy using 6 different devices in 4 anatomic positions: Clinical outcomes and technical considerations. $J$ Thorac Cardiovasc Surg 2015;150:1557-67

Clinical outcomes after transcatheter valve-in-valve therapy with different devices and in different positions. They show that there is a significant patient-prosthesis mismatch especially in valves smaller than $25 \mathrm{~mm}$.

[9] Barbanti M, Petronio AS, Ettori F, et al. 5-Year Outcomes After Transcatheter Aortic Valve Implantation With CoreValve Prosthesis. JACC Cardiovasc Interv 2015;8:1084-91

[10] Grant SW, Hickey GL, Ludman P, et al. Activity and outcomes for aortic valve implantations performed in England and Wales since the introduction of transcatheter aortic valve implantation. Epub: Eur J Cardiothorac Surg 2015(Aug), doi: 10.1093/ejcts/ezv270 [11] Bourguignon T, Bergöend E, Mirza A et al. Risk factors for valve-related complications after mechanical heart valve replacement in 505 patients with long-term follow up. J Heart Valve Dis $2011 ; 20: 673-80$ 
[12] Oxenham $\mathrm{H}$, Bloomfield $\mathrm{P}$, Wheatley DJ et al. Twenty year comparison of a Bjork-Shiley mechanical heart valve with porcine bioprostheses. Heart 2003;89:715-21

[13] Birkmeyer NJ, Birkmeyer JD, Tosteson AN et al. Prosthetic Valve Type for Patients Undergoing Aortic Valve Replacement: A Decision Analysis. Ann Thorac Surg 2000;70:194652

[14] Hammermeister K, Sethi GK, Henderson WG et al. Outcomes 15 Years After Valve Replacement With a Mechanical Versus a Bioprosthetic Valve: Final Report of the Veterans Affairs Randomized Trial. J Am Coll Cardiol 2000;36:1152-8

[15] Wieloch M, Själander A, Frykman V et al. Anticoagulation control in Sweden: reports of time in therapeutic range, major bleeding, and thrombo-embolic complications from the national quality registry AuriculA. Eur Heart J 2011;32:2282-9

[16] Heneghan C, Ward A, Perera R et al. Self-monitoring of oral anticoagulation: systematic review and meta-analysis of individual patient data. Lancet 2012;379:322-34

[17] Eikelboom JW, Connolly SJ, Brueckmann M et al. Dabigatran versus warfarin in patients with mechanical heart valves, NEJM 2013;369:1206-14

[18] Stewart S, Hart CL, Hole DJ, et al. Population prevalence, incidence, and predictors of atrial fibrillation in the Renfrew/Paisley study. Heart 2001;86:516-21.

[19] Heeringa J, van der Kuip D, Hofman A et al. Prevalence, incidence and lifetime risk of atrial fibrillation: the Rotterdam study. Eur Heart J 2006;27:949-53.

*20] Stassano P, Di Tommaso L, Monaco M et al. Aortic valve replacement: a prospective randomized evaluation of mechanical versus biological valves in patients ages 55 to 70 years. J Am Coll Cardiol 2009;54:1862-8

In a 13 year follow-up after AVR, they showed a slightly higher bleeding rate in patients with mechanical valves, but significantly higher rate of SVD and reoperation after AVR with bioprostheses. 
*[21] Brown ML, Schaff HV, Lahr BD, et al. Aortic valve replacement in patients aged 50 to 70 years: improved outcome with mechanical versus biologic prostheses. J Thorac Cardiovasc Surg 2008;135:878-884

Brown et al showed a survival advantage of patients receiving a mechanical AVR between 50 to 70 years of age.

[22] Weber A, Noureddine H, Englberger L et al. Ten-year comparison of pericardial tissue valves versus mechanical prostheses for aortic valve replacement in patients younger than 60 years of age. J Thorac Cardiovasc Surg. $2012 ; 144: 1075-83$

*[23] Glaser N, Jackson V, Holzmann MJ, et al. Aortic valve replacement with mechanical vs. biological prostheses in patients aged 50-69 years. Epub: Eur Heart J 2015(Nov), doi: 10.1093/eurheartj/ehv580

Patients aged 50-69 years who received mechanical valves had better long-term survival after AVR than those with bioprostheses.

[24] Habib G, Thuny F, Avierinos J. Prosthetic valve endocarditis: current approach and therapeutic option. Prog Cardiovasc Dis. 2008;50:274-81

[25] Brennan JM, Edwards FH, Zhao $\mathrm{Y}$ et al. Long-term safety and effectiveness of mechanical versus biologic aortic valve prostheses in older patients: results from the Society of Thoracic Surgeons Adult Cardiac Surgery National Database. Circulations 2013;127:164755

[26] Baddour LM, Wilson WR, Bayer AS et al. Infective endocarditis: diagnosis, antimicrobial therapy, and management of complications: a statement for healthcare professionals from the Committee on Rheumatic Fever, Endocarditis, and Kawasaki Disease, Council on Cardiovascular Disease in the Young, and the Councils on Clinical Cardiology, Stroke, and Caridovascular Surgery and Anesthesia, American Heart Association: endorsed by the 
infectious Diseases Society of America. Circulation 2005;111:394-434

[27] Nguyen DT, Delahaye F, Obadia JF et al. Aortic valve replacement for active infective endocarditis: 5-year survival comparison of bioprostheses, homografts and mechanical prostheses. Eur J Cardiothorac Surg 2010;37:1025-32

[28] Moon MR, Miller DC, Moore KA et al. Treatment of endocarditis with valve replacement: the question of tissue versus mechanical prosthesis. Ann Thorac Surg 2001;71:1164-71

[29] Massel DR and Little SH. Antiplatelet and anticoagulation for patients with prosthetic heart valves. Cochrane Database Syst Rev. 2013;7:CD003464

[30] Altman R, Rouvier J, Gurfinkel E et al. Comparison of two levels of anticoagulant therapy in patients with substitute heart valves. J Thorac Cardiovasc Surg 1991;101:427-31

*[31] Elkayam U, Bitar F. Valvular Heart Disease and Pregnancy. JACC 2005;46:403-10

Review of valvular heart disease in pregnancy. A good overview of problems in women of child bearing age (oral anticoagulation, risk of reoperation, accelerated SVD).

[32] Lawley CM, Lain SJ, Algert CS et al. Prosthetic heart valves in pregnancy, outcomes for women and their babies: a systematic review and meta-analysis. BJOG 2015;122:1446-55

[33] Task Force on the Management of Cardiovascular Diseases During Pregnancy of the European Society of Cardiology. Eur Heart J 2003;24:761-81

[34] Larrea JL, Nuñez L, Reque JA, et al. Pregnancy and mechanical valve prostheses: a high-risk situation for the mother and the fetus. Ann Thorac Surg 1983;36:459-63

[35] Mihaljevic T, Paul S, Leacche $\mathrm{M}$ et al. Valve replacement in women of childbearing age: influences on mother, fetus and neonate. J Heart Valve Dis 2005;14:151-7

[36] von Hagen IM, Roos-Hesselink JW, Ruys TP, et al. Pregnancy in Women With a Mechanical Heart Valve: Data of the European Society of Cardiology Registry of Pregnancy and Cardiac Disease (ROPAC). Circulation 2015;132:132-42 
[37] Chan V, Jamieson WRE, Fleisher AG, et al. Valve Replacement Surgery in End-Stage Renal Failure: Mechanical Prostheses Versus Bioprostheses. Ann Thorac Surg 2006;81:85762

[38] Boening A et Brück M. Heart valve prosthesis selection in patients with end-stage renal disease requiring dialysis: a systematic review and meta-analysis. Heart 2012;98:99

[39] Okada N, Tajima K, Takami Y, et al. Valve Selection for the Aortic Position in Dialysis Patients. Ann Thorac Surg 2015;99:1524-31

[40] Pai VB, Tai CK, Bhakri K, et al. Should we use mechanical valves in patients with endstage renal disease? Interact Cardioasc Thorac Surg 2012;15:240-3

[41] Puskas J, Gerdisch M, Nichols D, et al. Reduced anticoagulation after mechanical aortic valve replacement: interim results from the prospective randomized on-X valve anticoagulation clinical trial randomized Food and Drug Administration investigational device exemption trial. J Thorac Cadiovasc Surg 2014;147:1202-10

[42] Gallegos R, Rivard A, Suwan P, et al. In-vivo experience with the Triflo trileaflet mechanical heart valve. J Heart Valve Dis 2006;15:791-9

[43] Chan WS, Anand S, Ginsberg JS. Anticoagulation of pregnant women with mechanical heart valves: a systematic review of the literature. Arch Intern Med 2000;160:191-6

[44] Li T, Laí X, Bian C, Liu XH. Meta-analyses of pregnancy outcomes in women with mechanical heart valves treated by different anticoagulant regimens. J Evidence-based Med 2008;8:42-8

[45] Mol A, Smits A, Bouten C et al. Tissue engineering of heart valves: advances and current challenges. Expert Rev Med Devices. 2009;6:259-75.

[46] Driessen-Mol A, Emmert M, Dijkman P et al. Transcatheter implantation of homologous "off-the-shelf" tissue-engineered heart valves with self-repair capacity: long-term functionality and rapid in vivo remodeling in shee J Am Coll Cardiol. 2014 Apr 8;63:1320-9. 
Figure 1. Comparison between guidelines for the treatment of patients with valvular heart disease of the American Heart Association (AHA) / American College of Cardiology (ACC) 2014 and the European Society of Cardiology (ESC) 2012

\begin{tabular}{|l|l|l|l|l|l|l|}
\hline & \multicolumn{3}{|c|}{ AHA/ACC 2014 } & \multicolumn{3}{|c|}{ ESC 2012 } \\
\hline Guidelines & COR & LOE & Age & COR & LOE & Age \\
\hline $\begin{array}{l}\text { Choice of prosthetic valve type should be a shared } \\
\text { decision process, considering the desire of the informed } \\
\text { patient }\end{array}$ & I & C & & I & C & \\
\hline
\end{tabular}

ACC, American College of Cardiology; AHA, American Heart Association; COR, class of recommendation; LOE, level of evidence. 
Figure 2. On-X aortic heart valve (On-X Life Technologies, Inc.)
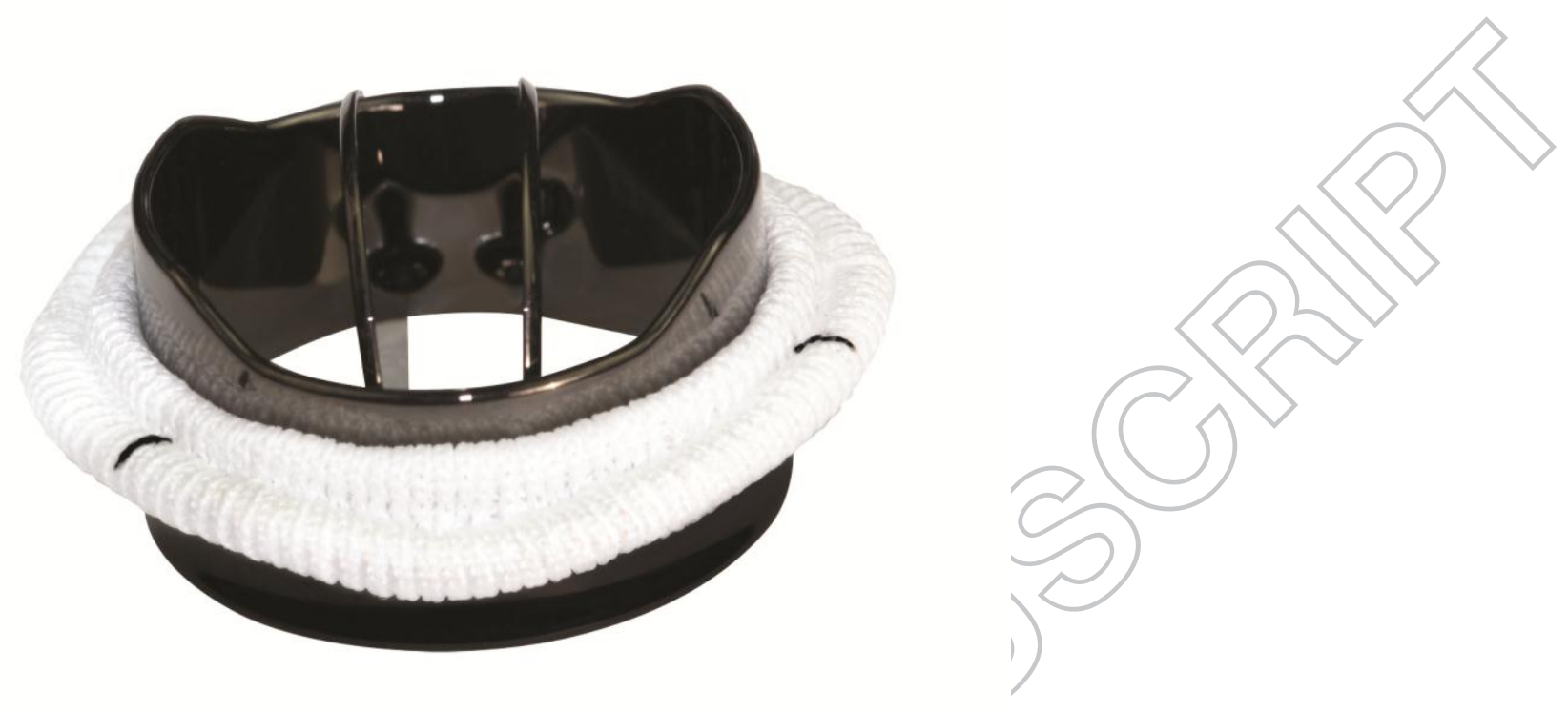
Figure 3. Lapeyre-Triflo FURTIVA ${ }^{\circledR}$

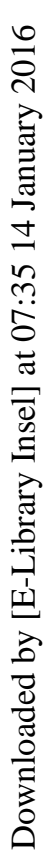

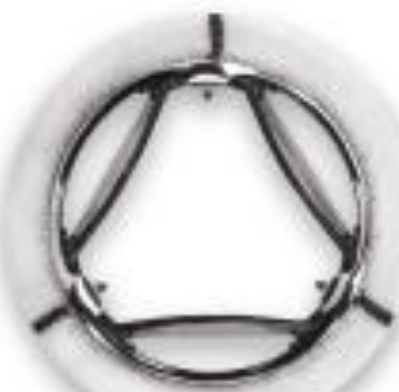

\section{Lapeyre-Triflo FURTIVA ${ }^{\circledR}$}

